Nota Científica

\title{
Paracrangon areolata Faxon, 1893 un camarón nuevo para Chile (Decapoda, Crangonidae)
}

\author{
Pedro Báez R. ${ }^{1}$ y Raúl Soto M. ${ }^{2}$ \\ ${ }^{1}$ Sección Hidrobiología, Museo Nacional de Historia Natural \\ Casilla 787, Santiago, Chile \\ ${ }^{2}$ Departamento de Ciencias del Mar, Universidad Arturo Prat \\ Casilla 121, Iquique, Chile
}

\begin{abstract}
RESUMEN: Se recolectaron 10 ejemplares de Paracrangon areolata Faxon en aguas chilenas: nueve entre $35^{\circ} 31^{\prime}$ S$73^{\circ} 04^{\prime} \mathrm{W}$ y $35^{\circ} 43^{\prime}$ 'S $-73^{\circ} 16^{\prime} \mathrm{W}$ y uno frente a Iquique. La distribución batimétrica de esta especie se determinó entre los 580 y $800 \mathrm{~m}$. Este hallazgo representa un género y una especie nueva para la fauna carcinológica de Chile, que vive en el talud continental integrando de la fauna arquibéntica.
\end{abstract}

Palabras claves: Decapoda, Pleocyemata, Crangonidae, Chile.

\section{Paracrangon areolata Faxon, 1893 a new shrimp from Chile (Decapoda, Crangonidae)}

\begin{abstract}
Ten specimens of Paracrangon areolata were collected in chilean waters: nine between $35^{\circ} 31^{\prime} \mathrm{S}-73^{\circ} 04^{\prime} \mathrm{W}$ and $35^{\circ} 43^{\prime} \mathrm{S}-73^{\circ} 16^{\prime} \mathrm{W}$ and one off Iquique. The bathymetric range of this species is between 580 and $800 \mathrm{~m}$. This finding represents a new genus and a new species to the crustacean fauna of Chile. These shrimps live on the continental slope and it integrates the Archibenthic fauna.
\end{abstract}

Key words: Decapoda, Pleocyemata, Crangonidae, Chile.

\section{INTRODUCCION}

La extensión de la pesca a profundidades cada vez mayores y la investigación científica del Talud Continental de Chile han permitido ir aumentando, en forma progresiva, el catastro de las especies que integran la fauna arquibéntica. Durante los últimos 15 años han sido numerosos los nuevos registros, de decápodos, (Retamal 1981 y 1994; Retamal y Soto, 1993). Con este hallazgo del camarón Paracrangon areolata la familia Crangonidae tiene tres especies en aguas de Chile.

\section{MATERIAL EXAMINADO}

Se revisaron diez ejemplares de los cuales nueve se recolectaron en pescas exploratorias de arrastre llevadas a cabo por el B/I "Itzumi" del Instituto de Fomento Pesquero de Chile (IFOP) el 28 de agosto de 1980 en el talud continental de Chile. Ocho están depositados en el Museo Nacional de Historia Natural (MNHNC-D) y corresponden a: Lance 80027 a 35 $31^{\prime} \mathrm{S}, 73^{\circ} 04^{\prime} \mathrm{W}, 620 \mathrm{~m}$ de profundidad: una hembra ovífera (MNHNC-D11146), una hembra (MNHNC-D11147) y un macho (MNHNCD11148); Lance 80029, a 35³8'S, 73²' 'W, 580 $m$ de profundidad: una hembra ovífera (MNHNCD11149); Lance 80030, a 35²43'S, 73¹6'W, 660 m de profundidad: un macho (MNHNC-D11150), y tres hembras ovíferas (MNHNC-D11151 al 11153). Un noveno ejemplar procede del Lance 80027 y corresponde a una hembra ovífera no catalogada. El décimo ejemplar es una hembra sin huevos capturada a comienzos de marzo de 1985, a unas 30 millas de la costa frente a Iquique, a $800 \mathrm{~m}$ de profundidad. Integraba la fauna acompañante en pescas comerciales de las centollas Paralomis longipes, Lithodes wiracocha y L. panamensis y está 
depositada en la colección carcinológica del Departamento de Ciencias del Mar de la Universidad Arturo Prat de Iquique.

\section{RESULTADOS}

\section{Descripción}

Es de cuerpo robusto con su tegumento carinado y esculturado. El caparazón está provisto de un rostro largo y agudo, fuertemente encorvado hacia arriba, comprimido lateralmente; con sus márgenes superior entero e inferior provisto de dos espinas, una cerca de la base del rostro, sobre los ojos y es larga; la otra más pequeña se halla cerca de su extremo distal. Una carina prominente, se continúa desde el rostro hacia atrás, a lo largo de la línea media dorsal del caparazón cefalotorácico; tiene cuatro espinas muy sobresalientes. La región branquial está dividida en numerosas celdas de variados tamaños, conformada por una trama formada por una serie de crestas unidas (Fig.1).

El borde inferior de la celda superior tiene tres espinas pequeñas; la anterior es la más grande. La región hepática es dilatada.

El abdomen está ornamentado con una carina baja, que es más conspicua sobre el tercer y sexto segmentos donde es doble. El telson es largo y termina en punta.

El primer par de periópodos es subquelado. Carece del segundo par. El tercer par es muy delgado y más largo que el primero. Los cuarto y quinto pares son robustos, y más largos que el tercero. El quinto par es más largo que el cuarto. Ambos terminan en un dáctilo encorvado y muy agudo.

\section{Datos somatométricos}

Los datos somatométricos de ejemplares machos (M) y hembras (H) examinados de Paracrangon areolata (en $\mathrm{mm}$ ), son los siguientes:

\section{COMENTARIOS}

La morfología de Paracrangon areolata se ajusta a la descripción de la especie dada por Méndez (1981). Sin embargo, uno de los ejemplares examinados, que lamentablemente no fue conservado, tenía en el rostro una espina supernumeraria, encorvada hacia arriba y hacia adelante (Figs. 2 y 3, señalada con una flecha).

Faxon (1895) señaló la distribución de Paracrangon areolata para $21^{\circ} 15^{\prime} \mathrm{N}, 106^{\circ} 23^{\prime} \mathrm{W}$ y $21^{\circ} 19^{\prime} \mathrm{N}$, 106²4'W, a 676-680 brazas (1237-1244 m). Del Solar (1972) amplió su distribución en el hemisferio sur hasta $13^{\circ} 47^{\prime} \mathrm{S}, 81^{\circ} 12^{\prime} \mathrm{W}$, a $650 \mathrm{~m}$ de profundidad y Méndez (1981) la extendió hasta $17^{\circ} 37^{\prime} \mathrm{S}$, 71'51'W y $800 \mathrm{~m}$ de profundidad.

Con estos hallazgos se amplía el límite sur de su distribución hasta $35^{\circ} 43^{\prime} \mathrm{S}$. En Chile su distribución batimétrica oscila entre 580 m y 800 m. La comparación con el rango de profundidad del material obtenido en el Perú y frente a México confirma que esta especie, como otras arquibénticas, se encuentra a profundidades cada vez mayores a medida que se avanza hacia latitudes ecuatoriales.

Con este hallazgo Crangonidae incorpora un género y una especie nueva para aguas chilenas y queda representada por: Paracrangon areolata Faxon 1893; Pontophylus occidentalis Faxon 1893 (Méndez, 1981) y Sclerocrangon atrox Faxon, 1893 (Bahamonde, 1981).

Paracrangon areolata es una especie arquibéntica que, sin embargo, podría ser considerada también batial o batiabisal. Según Andrade (1987) estas especies están bien adaptadas a vivir en aguas profundas, a temperaturas relativamente bajas, tanto en las zonas central y norte de Chile, como del Perú pueden alcanzar latitudes subecuatoriales en el Pacífico noreste.

\begin{tabular}{|lcccccccccc|}
\hline MEDIDAS / SEXO & $\mathbf{M}$ & $\mathbf{M}$ & $\mathbf{H}$ & $\mathbf{H}$ & $\mathbf{H}$ & $\mathbf{H}$ & $\mathbf{H}$ & $\mathbf{H}$ & $\mathbf{H}$ & $\mathbf{H}$ \\
$\mathbf{N}^{\mathbf{0}}$ DE CATALOGO & $\mathbf{1 1 1 4 8}$ & $\mathbf{1 1 1 5 0}$ & $\mathbf{1 1 1 4 6}$ & $\mathbf{1 1 1 4}$ & $\mathbf{1 1 1 4 9}$ & $\mathbf{1 1 1 5 1}$ & $\mathbf{1 1 1 5 2}$ & $\mathbf{1 1 1 5 3}$ & (Fig. 2) & Iquique \\
\hline Longitud total & 71,1 & $\mathbf{6 3 , 1}$ & 91,5 & 84,7 & 87,0 & 92,5 & 80,4 & 88,6 & 64,6 & 60,0 \\
\hline Longitud del rostro & 10,7 & 12,1 & 13,8 & - & 13,7 & 13,3 & 9,7 & 11,9 & 11,0 & 10,0 \\
\hline Longitud del caparazón & 27,2 & 25,6 & 33,4 & 26,9 & 32,7 & 32,7 & 26,9 & 30,6 & 23,9 & 24,0 \\
\hline Longitud del abdomen & 44,8 & 38,9 & 56,8 & 54,3 & 57,2 & 58,9 & 53,1 & 58,2 & 39,7 & 36,0 \\
\hline Ancho del caparazón & 12,3 & - & 15,7 & 15,0 & 21,1 & 26,0 & 21,8 & 21,5 & 12,7 & - \\
\hline
\end{tabular}




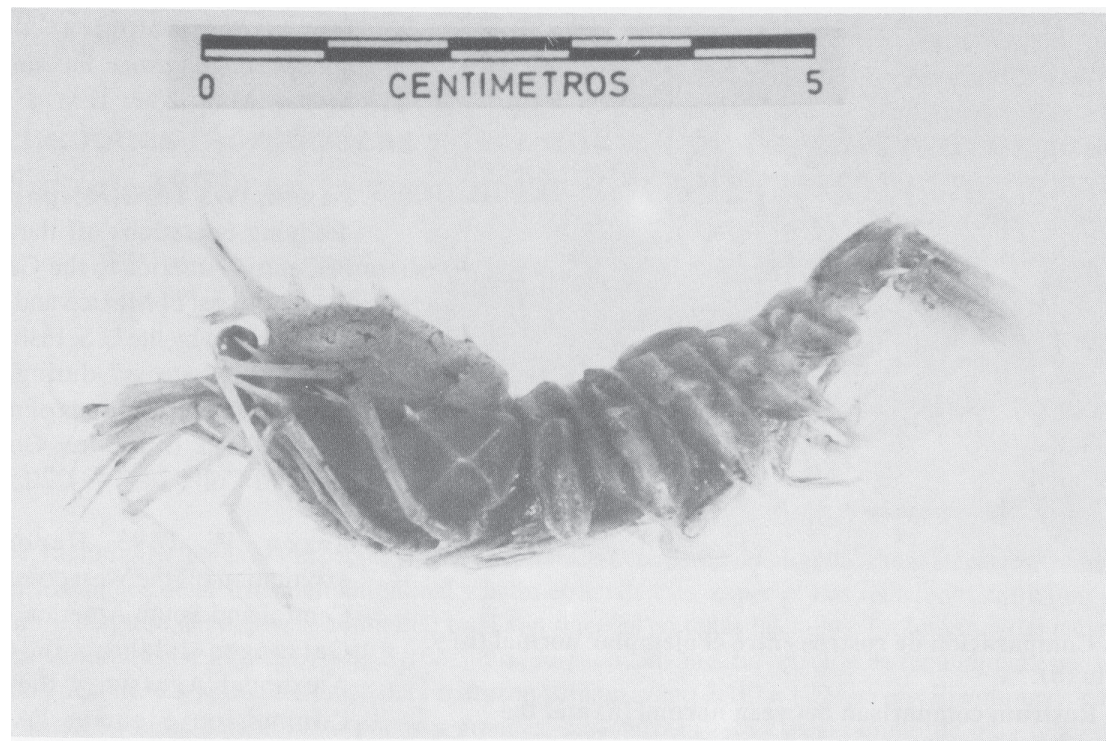

Figura 1. Vista lateral de Paracrangon areolata.

Figure 1. Lateral view of Paracrangon areolata.

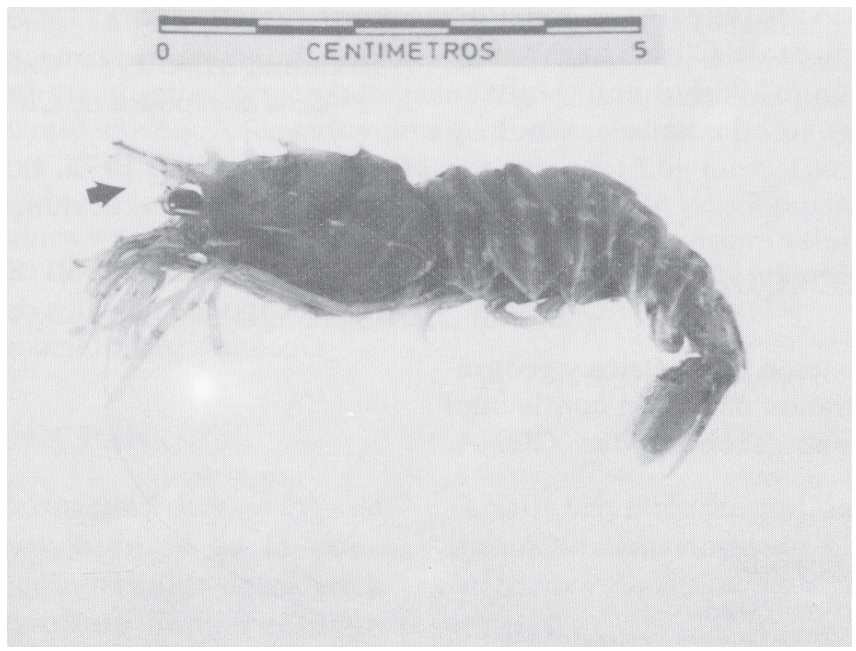

Figura 2. Paracrangon areolata hembra, con espina rostral-ventral supernumeraria (indicada con flecha). Figure 2. Paracrangon areolata female, with extra ventral rostral-spine (arrow). 


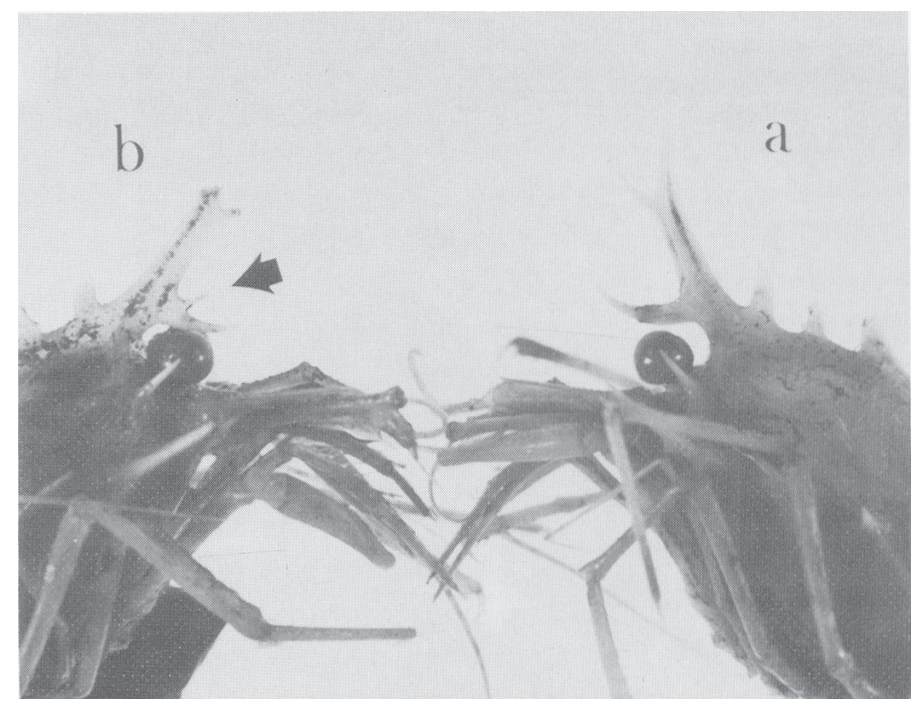

Figura 3. Comparación de rostros entre el ejemplar normal (a) y el anómalo (b).

Figure 3. Rostrum comparison between normal (a) and the anomalous (b) specimens.

\section{AGRADECIMIENTOS}

Los autores agradecen al Instituto de Fomento Pesquero de Chile y a los Profesores Gabriel Henríquez (Instituto de Fomento Pesquero), Ismael Kong (Universidad de Antofagasta) y Patricio Zavala (Universidad Católica de Chile) la recolección del material y al Dr. Ingo Werhtmann (Universidad Austral de Valdivia) su constante estímulo y sugerencias al manuscrito.

\section{REFERENCIAS}

Andrade, H. 1987. Distribución batimétrica y geográfica de macroinvertebrados del talud continental de Chile Central. Cienc. Tecnol. Mar, CONA, Valparaíso, 11: 61-94.

Recibido el 21 de junio de 1996. Aceptado el 14 de agosto de 1996.
Bahamonde, N. 1981. Adiciones a la fauna carcinológica de Chile: Sclerocrangon atrox Faxon 1893. Not. Mens., Mus. Nac. Hist. Nat., Chile, 25(296): 3-6.

Faxon, W. 1893. Reports on the dredging operations off the west coast of Central America to the Galapagos to the west coast of Mexico and in the Gulf of California by the U.S. Fish Comission Steamer Albatross" during 1891. VI. Preliminary descriptions of new species of Crustacea. Bull. Mus. Comp. Zool., Harvard Coll., 24: 149-220.

Faxon, W. 1895. Reports on an exploration off the West coast of Mexico Central and South America, and off the Galapagos Islands, in charge of Alexander Agassiz, y the U.S. Fish Commission Steamer "Albatross", during 1891, Lieut.-Commander Z.L. Tarmer U.S.N., commanding XV. The stalk eyed Crustacea. Mem. Mus. Comp. Zool., Harvard College 18: 5-292+66 Láms.

Méndez, M. 1981. Claves para la identificación y distribución de los langostinos y camarones (Crustacea Decapoda) del mar y ríos de la costa del Perú. Bol. Inst. Mar Perú-Callao, 5: 1-170.

Retamal, M.A. 1981. Catálogo ilustrado de los crustáceos decápodos de Chile. Gayana (zool.), 44: 1-110.

Retamal, M.A. 1992. Los Lithodidae chilenos. Ans. Ins. Pat., Punta Arenas (Chile), 21: 111-129.

Retamal, M.A. y R. Soto M. 1993. Crustáceos decápodos abisales de la zona Iquique-Arica. Est. Oceanol., Antofagasta, 12: 1-8. 